\title{
Impact of the Secondary Network on the Outage Performance of the Primary Service in Spectrum Sharing
}

\author{
Mohammad G. Khoshkholgh, Keivan Navaie \\ Department of Electrical and Computer Engineering \\ Tarbiat Modares University, Tehran, Iran \\ Email: navaie@modares.ac.ir
}

\author{
Halim Yanikomeroglu \\ Systems and Computer Engineering Department \\ Carleton University, Ottawa, Canada \\ Email: halim@sce.carleton.ca
}

\begin{abstract}
In this paper, we utilize stochastic geometry to analyze the primary service outage performance for spectrum sharing in Rayleigh fading environment. Using this approach, the impacts of the secondary service parameters as well as the wireless environment on the primary service outage probability are analyzed. We further obtain a closed form for the primary service outage probability based on the transmit power of the secondary service as well as miss detection probability of the spectrum sensing. Furthermore, we obtain the maximum secondary service transmitter node density for a given outage probability constraint of the primary service. We also derived an upper bound to the achievable capacity of the primary service. Simulations results confirm the analytical derivations.
\end{abstract}

\section{INTRODUCTION}

To improve the utilization of the allocated frequency bands, spectrum sharing as a new access method is proposed by the Federal Communications Commission (FCC) [1]. In this method, under certain conditions, a Secondary Service is able to access to a frequency band formally allocated to the Primary Service [2], [3]. Various schemes are proposed in the literature for spectrum sharing (see, e.g., [4] and references therein) where all of them are widely named as Dynamic Spectrum Access (DSA).

In DSA, the secondary service dynamically detects and makes use of the spectrum holes or white spaces [3]. White spaces are those parts of the spectrum allocated to the primary user which are under-utilized in some particular times and specific locations. The DSA methods are divided underlay and overlay spectrum sharing approaches. In this paper, our focus is on the overlay spectrum sharing which is also referred to as Opportunistic Spectrum Access (OSA) in the related literature [4].

In OSA the secondary service utilizes a spectrum sensing to detect the spectrum holes. If the spectrum condition is detected as 'idle' (i.e. the primary service is inactive) the secondary service is allowed to transmit its own information, otherwise, (i.e., the primary service is active), the secondary service is not allowed to make transmission. Therefore, given perfect spectrum sensing, adopting OSA not only increases the spectrum efficiency, but also imposes no negative impact on efficiency of the primary service.
However, perfect spectrum sensing is not possible in practice [3], [4]. The inaccuracy in the spectrum sensing, results in Quality of Service (QoS) degradation of the primary service users. Furthermore, by increasing the number of the secondary service users, the spectrum sensing inaccuracy, may seriously affect the performance of the primary network. Therefore, in spectrum sharing, it is essential to manage the secondary service parameters so that the primary service QoS constraints, such as the outage probability and achievable capacity, are always kept satisfied.

One of the main secondary service parameters to be adjusted is the maximum number of the secondary service users in a given coverage area of the primary network so that the primary service QoS parameters, e.g., outage probability is guaranteed. To obtain the maximum number of the secondary service users, in this paper, we employ stochastic geometry (see, e.g., [5]) which is shown to be a very powerful mathematical tool for performance evaluation of wireless networks (see, e.g., [6], [7].

Upper and lower bounds of the transmitter node density on a given plane to satisfy the outage probability constraint in Code Division Multiple Access (CDMA) ad hoc networks is obtained in [8] based on homogenous Poisson Point Process. The impact of fading, power allocation, and interference cancellation on the outage probability in ad hoc networks is also studied in [9] and [10].

In cognitive radio literature, stochastic geometry is also employed to analyze spectrum sharing performance. Channel capacity of the secondary service is the focus of [11]. Furthermore, in [12] distance-dependence path-loss attenuation model is considered and log-normal distribution is proposed as an approximated probability distribution function (PDF) of the aggregate interference at the primary service. Including the fading effects of the wireless channel as well as spectrum sensing parameters, [13] proposes an approximation for the interference aggregation distribution at the primary service receiver. They also analyze the impact of cooperative spectrum sensing among the secondary service users on the primary service outage probability. However, they assume that the secondary service transmitters is able to identify the spectrum 
status using signaling channels among the primary service receiver, and the secondary service transmitters. Furthermore, closed form for the primary service outage probability has not been proposed.

In this paper, utilizing stochastic geometry approach in modelling spectrum sharing system, we obtain a closed form formula for the primary service outage probability. In addition to the distance-dependent path-loss attenuation which is considered in the pervious works, we also consider fading in our modelling. In our modelling, we also consider inaccurate spectrum sensing. Inaccurate spectrum sensing is modelled through a receiver operating characteristic (ROC) curve which relates miss-detection and false alarm probabilities. For simplicity in our analysis we consider identical ROC curve for all secondary users in the coverage area. The maximum secondary service transmitters density is then obtained based on the outage probability constraint of the primary service as well as primary and secondary service parameters. We further obtain an upper bound on the achievable capacity of the primary service.

\section{SYSTEM MODEL}

\section{A. Channel Model}

The wireless channel is considered to be an Additive White Gaussian Noise (AWGN) with flat fading and noise power spectral density of $N_{0}$. The propagation power loss in this paper is modelled based on a loss function, $l(x, y)$, which returns the path-loss between locations $x=\left(x_{1}, x_{2}\right)$ and $y=\left(y_{1}, y_{2}\right), x, y \in \mathbb{R}^{2}$. In general, $l(x, y)$ is a function of $\|x-y\|$, where

$$
\|x-y\| \triangleq \sqrt{\left(x_{1}-y_{1}\right)^{2}+\left(x_{2}-y_{2}\right)^{2}} .
$$

In this paper, we consider distance-dependent path-loss, where $l(x, y)=\|x-y\|^{-\alpha}$ [7], [8]. Parameter $\alpha>2$ is the pathloss exponent. It most be noted that for $x=y, l(x, y) \rightarrow \infty$; however, since similar to [14], our main focus in this paper is to evaluate the probabilistic behavior of the network, this singularity does not have major effect on the obtained results.

In addition to the distance-dependent path-loss, small scale variations known as multi-path fading are also observed in a wireless channel. In this paper, we assume Rayleigh fading distribution with mean-value $1 / \mu$, i.e., $\sqrt{g}$ is Rayleigh where $f_{g}(g)=\mu e^{-\mu g} U(g)$.

\section{B. Spectrum Sharing and Spectrum Sensing}

Consider a single primary service transceiver where the receiver is located at the center. We assume that the distance between the primary transmitter and receiver is, $R>1$. The primary service transmitter transmits with a fixed power, $S_{p}$. The fading in the channel between the primary transmitter and receiver is, $g_{p}$. Therefore, the received power at the primary receiver is equal to $S_{p} R^{-\alpha} g_{p}$. The primary service accesses to the spectrum with probability $p_{b}$ which is the primary service activity. Therefore, the channel is idle with probability $p_{i}$, $p_{i}=1-p_{b}$.
The primary transmitter is surrounded by a secondary service ad hoc network. The secondary users acquire access to the spectrum by adopting OSA. Each secondary node senses the spectrum and if it is idle, the corresponding secondary service transmitter starts transmission. Transmission is stopped immediately in case transmission activity by the primary service is sensed by the secondary service. Recognizing the spectrum status is called spectrum sensing.

The spectrum sharing efficiency is significantly affected by the performance of spectrum sensing. The performance of the spectrum sensing is determined by two important parameters namely probability of false alarm, $\epsilon$, and probability of miss detection, $\delta,[4]$. If the spectrum is idle and the spectrum sensing wrongly recognize the spectrum status as busy, a false alarm is occurred. Furthermore, miss detection is occurred in cases where the spectrum is busy and it is mistakenly recognized as idle. On one hand, a spectrum sensing with a higher probability of false alarm results in a lower corresponding achieved secondary service capacity by limiting its access to the idle periods of the spectrum. On the other hand, a sensing procedure with a higher miss detection probability, results in interfering the primary service by the secondary transmission and increases its outage probability. Therefore, the ideal sensing procedure is the one with false alarm and miss detection probabilities equal to zero.

In practice however, the false alarm and miss detection probabilities are related to each other through a receiver operating characteristic (ROC) curve, which is a fundamental attribute of each spectrum sensing system [4]. The ROC curve usually presents the detection probability, i.e., $1-\delta$ versus $\epsilon$. In other words, for a given value of $\delta$, utilizing ROC curve, $\epsilon$ is obtained. Here, we for brevity of expositions we assume that the ROC curve is identical for all of the secondary service users. This assumption is not very accurate in practice. Indeed, the secondary service users those are located nearby the primary service, have higher spectrum sensing performance, i.e., lower miss detection probability.

Let the secondary service users be distributed on a plane based on a homogenous Poisson point process with density $\lambda_{s}$. Therefore, for a given region, $\mathcal{R}$, with area $A \mathrm{~m}^{2}$, the probability of having $k$ active $^{1}$ secondary service transmitters is obtained as

$$
\mathbf{P}\{k \text { in } \mathcal{R}\}=e^{-\lambda_{s} A} \frac{\left(\lambda_{s} A\right)^{k}}{k !} \quad k=1,2, \ldots
$$

Based on this spatial distribution model, the number of the secondary service transmitters, for two disjoint regions $\mathcal{R}_{1}$ and $\mathcal{R}_{2}$ are independent random variables [5]. In our modelling, similar to the previous works, e.g., [8], [12], the distance between a secondary service transmitter-receiver pair is assumed to be a fixed value. The secondary service transmission power is also assumed to be fix.

\footnotetext{
${ }^{1}$ The secondary service users which are sensing the spectrum and have traffic in the queue ready to be transmitted are referred to as active secondary service user.
} 


\section{The Primary Service Outage Probability}

In this section, we analyze the impacts of the secondary service users operation on the primary service performance. Particularly, our objective is to identify how the density of the secondary service transmitters affect the performance of the primary service. In our analysis the outage probability is considered as the primary service performance metric.

Miss detection in the secondary service spectrum sensing results in secondary service transmission during the activity period of the primary service. The secondary service transmission in such cases, imposes interference at the primary service receiver. By aggregating such interference at the primary service receiver, outage event may be occurred. Therefore, the aggregate interference at the primary service receiver is an important measure to model the impact of miss detection in spectrum sensing on the performance of the primary service.

\section{A. Aggregate Interference}

Assume that the primary receiver is located at the origin and surrounded by secondary users. Set $\tilde{\Pi}_{s}=\left\{X_{i}, i \geq 1\right\}$ is the Poisson point process with density $\lambda_{s}$ which indicates the location of the secondary service transmitters. Among secondary service transmitters some experience miss detection in their spectrum sensing.

Let $\breve{\Pi}_{s} \triangleq\left\{X_{i} \in \tilde{\Pi}_{s} \mid D_{i}=1, i \geq 1\right\}$ is a set including the location of the secondary service transmitters with miss detection, where $D_{i}=1\left(D_{i}=0\right)$ if the secondary service transmitter located at $X_{i}$ detects the spectrum status as idle (busy), while it is active.

Proposition 1: For a spectrum sharing system with miss detection probability of $\delta$ and primary service activity $p_{b}, \breve{\Pi}_{s}$ is a homogenous Poisson point process with density $\lambda$, where $\lambda=\delta p_{b} \lambda_{s}$, and $\lambda_{s}$ is the density of $\tilde{\Pi}$.

Corresponding to $\breve{\Pi}_{s}$, we define

$$
\Pi_{s} \triangleq\left\{\left(X_{i}, g_{s p, i}\right) \mid X_{i} \in \breve{\Pi}_{s}, i \geq 1\right\},
$$

which corresponds $X_{i} \in \breve{\Pi}_{s}$ with $g_{s p, i}$, where $g_{s p, i}$ is the fading of the wireless channel between the secondary transmitter located at $X_{i}$, and the primary receiver located at the origin. Set $\Pi_{s}$ is a homogenous Poisson point process over $\mathbb{R}^{2} \times[0, \infty) \times\{0,1\}$ with density $\lambda f_{g}\left(g_{s p}\right)$.

Transmission made by the secondary service transmitters located at $X_{i} \in \breve{\Pi}_{s}, i=1,2, \ldots$ imposes an aggregate interference, $I_{\phi}$, at the primary service receiver, where

$$
I_{\phi}=\sum_{\left(X_{i}, g_{s p, i}\right) \in \Pi_{s}} S_{s} u_{i}^{-\alpha} g_{s p, i} .
$$

In (2), $u_{i}=\left\|X_{i}\right\|$ is the distance between the secondary service transmitter located at $X_{i}$, and the origin.

\section{B. The Outage Probability}

The signal to interference plus noise ratio (SINR) at the primary service receiver is

$$
\operatorname{SINR}_{p}=\frac{S_{p} R^{-\alpha} g_{p}}{W+I_{\phi}}
$$

where $I_{\phi}$ is the aggregate interference, (2), and $W$ is a random variable represents the AWGN noise with variance $\sigma_{W}^{2}=N_{0} B$. The outage probability of the primary service user is defined as

$$
P_{\text {out }}^{p}=\mathbf{P}\left\{\operatorname{SINR}_{p}<\gamma_{t h}\right\},
$$

where $\gamma_{t h}$, is the SINR threshold. The outage may be caused by high interference $I_{\phi}$ and/or deep fading (i.e., very low $g_{p}$ ) between the primary transmitter and receiver ${ }^{2}$. In the following proposition we obtain $P_{\text {out }}^{p}$.

Proposition 2: The outage probability of the primary service is

$$
P_{\text {out }}^{p}=1-\Phi_{W}\left(\mu \frac{\gamma_{t h} R^{\alpha}}{S_{p}}\right) e^{-\lambda R^{2}\left(\frac{S_{s} \gamma_{t h}}{S_{p}}\right)^{\frac{2}{\alpha}} L},
$$

where $L$ is defined as

$$
L=\frac{2 \pi}{\alpha} \Gamma\left(\frac{2}{\alpha}\right) \Gamma\left(1-\frac{2}{\alpha}\right),
$$

$\Gamma(x)=\int_{0}^{\infty} t^{x-1} e^{-t} d t$, and $\Phi_{W}(s)$ is the Laplace transform of the pdf of AWGN.

In practice, the outage probability in the primary service requires to be always less than or equal to $\xi$, which is a system parameter, i.e., $P_{\text {out }}^{p} \leq \xi$. As it is seen in Proposition 2, different factors affect the outage probability. Here, our main focus is to study the effect of $\lambda_{s}$, on the outage probability of the primary user. For the case that, $W \ll I_{\phi}$ and a given set of system settings, the following proposition provides the maximum secondary service node density, $\lambda_{s}^{*}$, where the outage probability constraint is held.

Proposition 3: If $W \ll I_{\phi}$, the maximum secondary service transmitter density, $\lambda_{s}^{*}$, while $P_{\text {out }}^{p} \leq \xi$, is

$$
\lambda_{s}^{*}=\left(\frac{S_{s} \gamma_{t h}}{S_{p}}\right)^{\frac{-2}{\alpha}} \frac{R^{-2}}{L \delta p_{b}} \ln \frac{p_{b}}{p_{b}-\xi}
$$

where $L$ is defined as in (6).

Proposition 3 gives the closed form formula for the maximum allowable secondary service transmitter density. Proposition 3 furthermore suggests that for a given primary service outage constraint, $\xi$, the appropriate density of the secondary service transmitters, $\lambda_{s}$, is related to the wireless channel condition, represented by $L$, the primary service parameters, such as $S_{p}, R$, and $\gamma_{t h}$, and the primary service activity $p_{b}$.

Furthermore, the secondary service can also adjust $S_{s}$ and $\delta$ so that the outage capacity constraint is held. As it is seen, by decreasing miss detection probability $\delta, \lambda_{s}^{*}$ is also increased. Thus, the secondary service can adopt a more accurate sensing procedure with a lower miss detection probability and/or reduce its transmission power.

In the following we also elaborate on another important criteria for the primary service which is its capacity in presence

\footnotetext{
${ }^{2}$ In some references in the literature, e.g., [13] it is assumed that the primary service receiver is sensitive to the amount of $I_{\phi}$. If $I_{\phi}$ is larger than a given interference threshold, $I_{t h}$, outage is occurred. Our definition here is more tractable in practice.
} 
TABLE I

Simulation PARAMETERS

\begin{tabular}{l|c}
\hline Parameter & Value \\
\hline Propagation loss exponent & 4 \\
Cell radius & $5000 \mathrm{~m}$ \\
$R$ & $20 \mathrm{~m}$ \\
$\mu$ & $8 \mathrm{~dB}$ \\
Background noise power $\left(N_{0} B\right)$ & $10^{-8} \mathrm{Watt}$ \\
$S_{s}$ & $0.1 \mathrm{Watt}$ \\
$S_{p}$ & $1 \mathrm{Watt}$ \\
$\gamma_{t h}$ & $10 \mathrm{~dB}$ \\
$p_{b}$ & 0.7 \\
\hline
\end{tabular}

of the aforementioned secondary transmission and provide an upper bound to the achievable capacity of the primary service.

\section{An Upper Bound on the Achievable Capacity of the Pri- mary Service}

Consider an AWGN channel with signal to noise ratio $\beta$ at the front-end of the receiver. For such communication channel, Shannon's capacity of the channel is equal to $1 / 2 \log (1+\beta)$ symbol per channel usage. In fading environments, because of random variations of channel gain, the channel capacity is obtained by averaging Shannon's capacity over all fading realizations which is called channel Ergodic capacity.

In our formulation, the SINR at the primary service receiver for a given fading condition is a random variable which is presented in (3). Therefore, the achievable Ergodic capacity of the primary service is

$$
C_{p}=\frac{1}{2} \mathbf{E} \log \left(1+\operatorname{SINR}_{p}\right),
$$

here $\mathbf{E}$ is expectation operator over the random variable $\mathrm{SINR}_{p}$. An upper bound to $C_{p}$ is provided in the following proposition.

Proposition 4: An upper bound to the achievable capacity of the primary service for $W \ll I_{\phi}$ is

$$
C_{p} \leq \frac{1}{2} \log \left(1+\frac{S_{p}}{S_{s}}\left(\frac{1}{\lambda L R^{2}}\right)^{\frac{\alpha}{2}} \Gamma\left(\frac{\alpha}{2}+1\right)\right) .
$$

\section{Simulation Results}

In this section simulation results are compared with the obtained analytical derivations in this paper. In our simulations we adopt Monte-Carlo method with sufficient number of iterations. The main simulation parameters are presented in Table I. The primary receiver is fixed at the origin. The number of secondary users in the secondary network is the output of Poisson distribution with mean value $\lambda_{s}$. Secondary users are uniformly distributed on a plane. To highlight the difference between the mathematical derivations and simulation results, in our simulations, the background noise power is considered higher than usual.

1) Primary Service Outage Probability: Fig. 1 presents the primary service outage probability versus the secondary service transmitters density, $\lambda_{s}$. Here, we assume that all the secondary service transmitters have the same ROC curve, we further fix $\delta=0.05$. In case of no secondary service transmitter, Fig. 1 indicates that, the outage probability of the

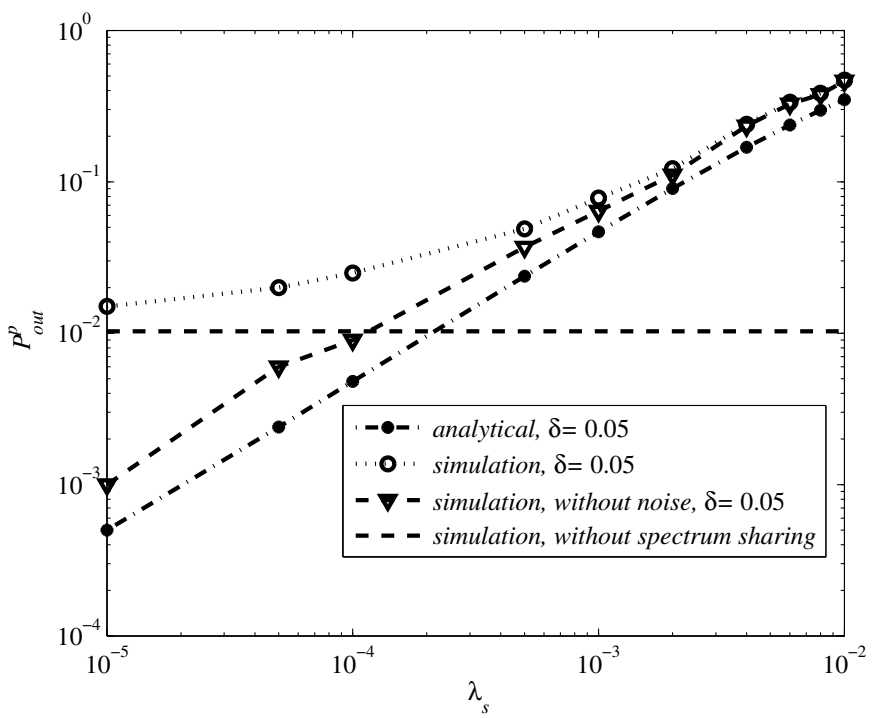

Fig. 1. The primary service outage probability $v s$. the density of the secondary service transmitters, $\lambda_{s}$, for $\delta=0.05, N_{0} B=0,10^{-8}$

primary service remains constant by increasing $\lambda_{s}$. As it is expected, in the presence of a secondary service network, it is also seen that by increasing $\lambda_{s}$, the outage probability of the primary service is also increased. Indeed, by increasing $\lambda_{s}$, the number of the secondary service transmitters with miss detection per unit area is increased.

In Fig. 1, we also compare the analytical results with the simulation results. As it is seen, by considering the background noise power, there is a gap between the simulations and analytical results. This gap is mostly eliminated when $\lambda_{s}$ is increased. This is mainly due to considering a higher than usual background noise in the simulation. Note that in the derivations the background noise was ignored. The gap is decreased since for higher $\lambda_{s}$, the share of the background noise in the total interference is decreased.

For the case where there is no spectrum sharing, it is seen that the analytical result is below the actual $P_{\text {out }}^{p}$. This is mainly due to ignoring the background noise. Therefore, this figure suggests that ignoring the background noise is acceptable only when the amount of $\lambda_{s}$ is sufficiently high. It must be noted that, here a disk with radius 5000 meter is considered, however, the analytical derivations are obtained for $\mathbb{R}^{2}$, i.e., infinite radius. This is the reason that there is some differences between the analytical and simulation results for higher values of $\lambda_{s}$. We also conduct simulation without considering the background noise, as it is seen in Fig. 1, in this case the analytical results follow the simulations closely.

2) Achievable Capacity of the Primary Service: In Fig. 2 the achievable capacity of the primary service is plotted versus $\lambda_{s}$. In this figure, for comparison we also present the achievable capacity of the primary service with no secondary service transmission.

As it is seen, in spectrum sharing, by increasing $\lambda_{s}$ the achievable capacity of the primary service is decreased. In this 


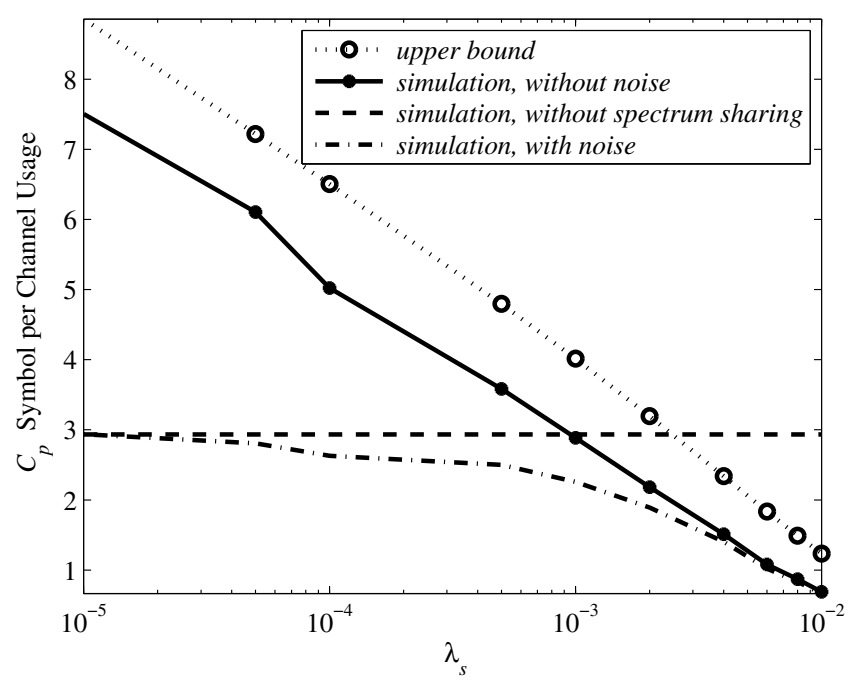

Fig. 2. The achievable capacity of the primary service $v s$. the secondary service transmitter density.

figure we also present the obtained analytical upper bound of the primary service capacity. It is seen that, the analytical and simulation results without considering the noise power have the same pattern; however, there is a gap between the obtained upper bound and the simulation results. The gap is decreased by increasing $\lambda_{s}$.

The gap, especially in the case of large enough $\lambda_{s}$, is partially because of the nature of Jensen's inequality. However, it must be noted that in presence of the background noise, the gap between the obtained upper bound and the achievable capacity for small enough $\lambda_{s}$ is very large. This is mainly due to ignoring the noise power in the derivations, although in such cases the power of the background noise is comparable with the aggregate interference. By increasing $\lambda_{s}$, the obtained upper bound and simulation result are very close. It is also observed in Fig. 2 that for small enough $\lambda_{s}$ the analytical result and simulation result without noise power have larger value respect to the system without spectrum sharing. This is mainly due to ignoring the noise power.

\section{CONCLUSIONS}

In this paper, we utilized stochastic geometry to analyze the primary service outage performance for spectrum sharing in Rayleigh fading environment. Using this approach, the impacts of the secondary service parameters and wireless environment on the primary service outage probability were analyzed. We further obtained a closed form for the primary service outage probability. The maximum secondary service transmitter node density for a given outage probability constraint of the primary service is then calculated. The obtained maximum density of the secondary service transmitters extends our vision on the essential secondary service parameters that adjusting them enables us to guarantee the performance of the primary service. We also presented an upper bound of the achievable capacity of the primary service.

\section{REFERENCES}

[1] "Spectrum policy taskforce report," Federal Communications Commission, Tech. Rep., November 2002, [online] Available: http://hraunfoss.fcc.gov/edocs_public/attachmatch/DOC-228542A1.pdf.

[2] J. M. Peha, "Approaches to spectrum sharing," IEEE Communications Magazine, pp. 10-12, February 2005.

[3] S. Haykin, "Cognitive radio: brain-empowered wireless communications," IEEE Journal on Selected Areas in Communications, vol. 23, no. 2, pp. 201-220, February 2005.

[4] Q. Zhao and B. Sadler, "A survey of dynamic spectrum access: Signal processing, networking, and regulatory policy," IEEE Signal Processing Magazine, vol. 24, no. 3, pp. 79-89, May 2007.

[5] J. F. C. Kingman, Poisson Processes. Oxford University Press, 1993.

[6] E. S. Sousa and J. A. Silvester, "Optimum transmission ranges in a direct-sequence spread-spectrum multihop packet radio network," IEEE Journal on Selected Areas in Communications, vol. 8, no. 5, pp. 762771, January 1990.

[7] F. Baccelli, B. Baszczyszyn, and P. Muhlethaler, "An ALOHA protocol for multihop mobile wireless networks," IEEE Transactions on Information Theory, vol. 52, no. 2, pp. 421-436, February 2006.

[8] S. P. Weber, X. Yang, J. G. Andrews, and G. de Veciana, "Transmission capacity of wireless ad hoc networks with outage constraints," IEEE Transactions on Information Theory, vol. 51, no. 12, pp. 4091-4102, December 2005.

[9] S. P. Weber, J. G. Andrews, and N. Jindal, "The effect of fading, channel inversion, and threshold scheduling on Ad Hoc networks," IEEE Transactions on Information Theory, vol. 53, no. 11, pp. 4127-4149, November 2007.

[10] S. P. Weber, J. G. Andrews, X. Yang, G. de Veciana, and N. Jindal, "Transmission capacity of wireless Ad Hoc networks with successive interference cancellation," IEEE Transactions on Information Theory, vol. 53, no. 8, pp. 2799-2814, August 2007.

[11] D. Zhang, Z. Tian, and G. Wei, "Spatial capacity of narrowband vs. ultra-wideband cognitive radio systems," IEEE Transactions on Wireless Communications, vol. 7, no. 11, pp. 4670-4680, November 2008.

[12] R. Menon, R. M. Buehrer, and J. H. Reed, "On the impact of dynamic spectrum sharing techniques on legacy radio systems," IEEE Transactions on Wireless Communications, vol. 7, no. 11, pp. 4198-4207, November 2008.

[13] A. Ghasemi and E. S. Sousa, "Interference aggregation in spectrumsensing cognitive wireless networks," IEEE Journal of Selected Topics in Signal Processing, vol. 2, no. 1, pp. 41-56, February 2008.

[14] A. M. Hunter, J. G. Andrews, and S. Weber, "Transmission capacity of ad hoc networks with spatial diversity," IEEE Transactions on Wireless Communications, vol. 7, no. 12, pp. 5058-5071, December 2008. 